\title{
Problems on rigidity of group actions and cocycles
}

\author{
S. HURDER \\ Mathematical Sciences Research Institute, 2223 Fulton Street, Berkeley, CA 94720, \\ USA
}

(Received 26 December 1984 and revised 15 February 1985)

\begin{abstract}
A conference on the interaction of ergodic theory, differential geometry and the theory of Lie Groups was held at the Mathematical Sciences Research Institute from May 24 to June 1, 1984. This is a report of the problem session organized by A. Katok and R. Zimmer and held on May 25, 1984 dealing with the topics in the title. Another problem session was centred on the rigidity of manifolds of non-positive curvature and related topics concerning their geodesic flows. This is reported on by $\mathrm{K}$. Burns and A. Karok separately [2].
\end{abstract}

The list of problems given here was compiled from those presented at the session and from suggestions made during the conference, and later refined by comments from the participants. A name next to a problem indicates that this person proposed it during the conference. Some of the problems given below are venerable, but many others reflect the current state of affairs in the topic at hand.

These notes, together with those of $\mathrm{K}$. Burns, are to be considered as a sequel to the problem list compiled by R. Spatzier at the A.M.S. Meeting at Amherst, October 1981 [29].

The problems are organized as follows, which is roughly the order of their presentation:

(1) Rigidity of smooth actions of lattices and Kazhdan groups.

(2) Rigidity of smooth actions of discrete subgroups of 'soft' Lie groups and horocycle flows.

(3) Rigidity of foliations.

(4) Classification of smooth cocycles and rigidity.

(5) $\varepsilon$-rigidity and $\varepsilon$-classification of cocycles.

(6) Rigidity and $\varepsilon$-rigidity of cocycles associated to smooth distal group actions.

(7) $L^{1}$-classification of cocycles.

(8) Miscellaneous problems on ergodic theory in differential geometry.

A reference beside an italicized word indicates where the appropriate definition may be found.

1. A basic problem is to construct non-algebraic examples of smooth volumepreserving actions of 'large' groups (e.g. lattices in semi-simple groups of higher 
rank) on manifolds of finite volume. Conversely, given a specific manifold one might try to show that all such actions must be algebraic.

Problem 1 (Zimmer). Are all volume-preserving analytic actions of SL $(n, \mathbb{R})$ for $n \geq 3$ describable in algebraic terms? Same question for a lattice in $\operatorname{SL}(n, \mathbb{R})$ ? For results along these lines, see [35].

Problem 2 (Zimmer). Can the natural action of $\operatorname{SL}(n, \mathbb{Z})$ on the $n$-torus $\mathbb{T}^{n}$, for $n \geqslant 3$, be perturbed to a non-algebraic action? Suppose an action of $\operatorname{SL}(n, \mathbb{Z})$ on $\mathbb{T}^{n}$ preserves an affine connection (cf. [37]). Does this imply the action is algebraic?

Remark (Mostow). One possible approach to problem 1 is to consider groups $\Gamma$ which decompose into a free product, or more generally an amalgamated product of finite groups and then construct for each of the finite groups an exotic action on the Euclidean sphere $S^{n} \subset \mathbb{E}^{n+1}$ which is not conjugate to a linear action. Such exotic actions of finite groups exist by the work of Cappell and Shaneson [3]. One difficulty to be overcome is that while each finite group preserves a volume on $\mathbb{E}^{n+1}$, an invariant volume for their free product must be constructed.

Remark (Weissfeiler). Millson and Johnson [21] have constructed perturbations of lattices in SO $(n, 1)$ which are discrete but not algebraic (so they must have infinite covolume by rigidity.) Possibly these can be used as a starting point for constructing non-algebraic actions on a manifold of finite volume.

Problem 3 (Fried). Show the action of $\operatorname{SL}(n, \mathbb{Z})$ on $\mathbb{T}^{n}$ is structurally stable [28].

Note that for $\gamma \in \mathrm{SL}(n, \mathbb{Z})$ hyperbolic, the action of $\gamma$ on $\mathbb{T}^{n}$ is structurally stable, so this may give an approach to a proof.

Problem 4 (Zimmer). There is a natural action of $\operatorname{SL}(n, \mathbb{Z})$ on the real projective space $\mathbb{R} P^{n-1}$. Does this action admit perturbations to non-algebraic actions? Note that the action in this case is not volume preserving.

A group $\Gamma$ is Kazhdan if the trivial representation is isolated in the left regular representation of $\Gamma$. For further discussions, see [32], [37].

Problem 5 (Zimmer). Every sufficiently small perturbation of an isometric action of a Kazhdan group $\Gamma$ must leave a smooth metric invariant [35]. Therefore, every sufficiently small perturbation of the action acts through the compact group of isometries of the corresponding invariant metric. Are such perturbations structurally stable? That is, does the compact group in question depend only upon $\Gamma$ and the tnanifold on which $\Gamma$ acts, or does it vary with the perturbation?

2. A group $\Gamma$ is soft if it is not expected to have any rigidity property. For example, a lattice in a semi-simple Lie group of rank $\geq 2$ is not soft, nor is $\Gamma$ having Kazhdan's Property T. Lattices in solvable groups are soft, as well as lattices in $\operatorname{SL}(2, \mathbb{R})$. For a soft group $\Gamma$, the relevant rigidity problems often involve the ergodic flows defined on the compact manifolds $X / \Gamma$, where $X$ is simply connected. These vague notions are made precise by the following remarks, results and problems.

Remark (Mostow). Let $\Gamma_{g}=\pi_{1}\left(\Sigma_{g}\right)$ be the fundamental group of the compact orientable surface of genus $g$. Each metric of constant negative curvature on $\Sigma_{g}$ 
determines an action of $\Gamma_{g}$ on $S^{1}$ via the action on the Poincare disc. All such actions of $\Gamma_{g}$ on $S^{1}$ are topologically conjugate, but if the actions are smoothly conjugate, then the metrics must be equivalent. In this case, the problems of smooth rigidity and topological rigidity are strikingly different.

As an example of a rigidity problem arising in this context, one can ask whether new $C^{\infty}$-actions of $\Gamma_{g}$ on $S^{1}$ can be obtained by using a metric $h$ on $\Sigma_{g}$ which has negative curvature, but it is not constant. Such a metric yields an action of $\Gamma_{g}$ on the hyperbolic disc, and the induced action on the boundary is always $C^{1}$. The following rigidity theorem is due to E. Ghys.

THEOREM [11]. Let $\psi: \Gamma_{g} \rightarrow \operatorname{PSL}(2, \mathbb{R}) \subseteq \operatorname{Diff}^{r}\left(S^{1}\right)$ be the representation coming from a metric with curvature -1 . Let $\psi^{\prime}: \Gamma_{g} \rightarrow \operatorname{Diff}^{r}\left(S^{1}\right)$ for $2 \leq r \leq \omega$ be another representation which is $C^{2}$-close to $\psi$. Then there is a diffeomorphism $h$, of class $C^{r-2}$, such that $h \psi^{\prime} h^{-1}$ consists of projective transformations in PSL $(2, \mathbb{R})$.

For $r=2$, this result is just the fact that $\psi$ is structurally stable.

Problem 6. Can the conclusion of the theorem above be improved to obtain $h$ is $C^{r-1}$ ? In particular, for a $C^{2}$-perturbation $\psi^{\prime}$ of $\psi$, is it $C^{1}$-conjugate to $\psi$ ?

Let $M$ be the unit tangent bundle of a surface $\Sigma_{g}$ with negative curvature. Let $\xi$ denote the geodesic flow on $M$ and $\eta$ the horocycle flow [24]. If the curvature of the metric is constant, then we can metrically identify $M=\hat{\Gamma}_{\mathrm{g}} \backslash \mathrm{SL}(2, \mathbb{R})$, where $\operatorname{SL}(2, \mathbb{R})$ has the normalized left-invariant metric which descends to the given metric on $\Sigma_{g}=\Gamma_{g} \backslash S L(2, \mathbb{R}) / \mathrm{SO}_{2}$. The group $\hat{\Gamma}_{g}$ fits into an extension $1 \rightarrow Z \rightarrow \hat{\Gamma}_{g} \rightarrow \Gamma_{g} \rightarrow 1$. The flow $\xi_{t}$ on $M$ is defined by right multiplication with

$$
\left(\begin{array}{cc}
e^{t} & 0 \\
0 & e^{-t}
\end{array}\right)
$$

on $\hat{\Gamma}_{g} \backslash S L(2, \mathbb{R})$ and $\eta_{t}$ is defined by multiplication with

$$
\left(\begin{array}{ll}
1 & 0 \\
t & 1
\end{array}\right)
$$

For measure spaces $(X, \mu)$ and $(Y, \nu)$ a flow $\varphi$ on $X$ is measurably conjugate [24] to a flow $\psi$ on $Y$ if there exists a measure preserving bijection $\Phi: X \rightarrow Y$ such that $\psi_{t} \circ \Phi(x)=\Phi \circ \varphi_{t}(x)$ for all $t$ and a.e. $x \in X$.

M. Ratner proved a rigidity theorem for the horocycle flow which became a starting point for many other developments.

THEOREM [24]. Suppose $M_{1}$ and $M_{2}$ are the unit tangent bundles associated to surfaces $\Sigma_{g_{1}}$ and $\Sigma_{g_{2}}$ with constant negative curvature. Suppose the horocycle flow $\eta_{1}$ on $M_{1}$ is measurably conjugate to the horocycle flow $\eta_{2}$ on $M_{2}$. Then $g_{1}=g_{2}$, and there exists an isometry $\Phi: \Sigma_{g_{1}} \rightarrow \Sigma_{g_{2}}$ inducing the conjugation.

There is more than one natural parametrization for the horocycles on a surface of variable negative curvature. Accordingly the horocycle flow may be defined differently. Let us fix the Margulis parametrization which is expanded uniformly by the geodesic flow (cf. [8]). 
Problem 7 (Feldman, Ornstein). Generalize Ratner's theorem to horocycle flows with Margulis parametrization on surfaces of variable negative curvature.

Feldman and Ornstein [8] proved that the measurable conjugacy of the horocycle flows implies $C^{1}$ conjugacy of geodesic flows. However, it is not known whether the conjugating diffeomorphism between the tangent bundles can always be modified to be generated by an isometry between the surfaces. See $([2, \S 3])$ for a discussion of questions related with this problem.

In his thesis [31], D. Witte generalized Ratner's theorem to the case of two flows $\psi_{i}, i=1,2$, on quotients $\Gamma_{i} \backslash G_{i}$ of semi-simple Lie groups, where $\psi_{i}$ is defined by right multiplication with a unipotent 1-parameter subgroup of $G$. The key point is that each flow is affine and has zero entropy.

Problem 8 (Witte). Let $G$ be a connected Lie group and $\Lambda \subset G$ a lattice. For $g \in G$ let $\psi_{g}$ denote the flow induced on $\Lambda \backslash G$ by right multiplication with a 1-parameter subgroup of $G$ containing $g$. Show that if $\psi_{g}$ is an ergodic action with zero entropy, then the action on $\Lambda \backslash G$ is rigid. That is, if $G^{\prime}, \Lambda^{\prime}, \psi_{\mathrm{g}}^{\prime}$ is another such system with $\left(\Lambda \backslash G, \psi_{\mathrm{g}}\right)$ measurably conjugate to $\left(\Lambda^{\prime} \backslash G^{\prime}, \psi_{\mathrm{g}^{\prime}}^{\prime}\right)$ then there is an algebraic isomorphism between them.

A factor of a flow $\varphi$ on a measure space $(X, \mu)$ is a flow $\psi$ on a measure space $(Y, \nu)$ and a measure-class preserving map $\Phi: X \rightarrow Y$ such that $\Phi \circ \varphi_{t}(x)=\psi_{t} \circ \Phi(x)$ for all $t$ and a.e. $x \in X$. If $\Phi$ has a measurable inverse, then $\Phi$ is a trivial factor.

For the horocycle flow $\eta$ on a surface $\Sigma_{g}$ with constant negative curvature, Ratner has classified the factors of $\eta$ by showing they are all algebraic [25]. This implies that $(Y, \psi)$ is a factor of $(M, \eta)$ if and only if $Y$ is the unit tangent bundle of a surface $\Sigma_{g^{\prime}}$ where $\Sigma_{g}$ covers $\Sigma_{g^{\prime}}$ and the flow on $Y$ is the horocycle flow.

Ratner has posed the problem of whether the factor theorem can be extended to manifolds with variable negative curvature [25, p. 283] and Feldman raised a related question at the conference.

Problem 9. Classify factors of the horocycle flow on a surface of variable negative curvature. Specifically, show that all such factors are discrete and come from isometric symmetries of the surface.

One approach to this problem is to follow Ratner's proof for the constant curvature case: First prove that any factor is discrete and the factor-space has the natural structure of a manifold. Then prove that a factor of the horocycle flow is also a factor of the geodesic flow. This requires an analogue of the Feldman-Ornstein result, namely to show that the projection map is continuous and projects geodesics into geodesics. The remaining steps are similar to problem 7 .

3. Gromov gave a remarkable proof of the Mostow rigidity theorem for cocompact lattices in $\mathrm{SO}(n, 1)$ acting on $\mathbb{H}^{n}=\mathrm{SO}(n, 1) / \mathrm{SO}_{n}$ which used properties of bounded cohomology groups [12] and ideal simplices in $\mathbb{H}^{n}$ to deduce that measurable maps given on the Furstenberg boundary of $\mathrm{SO}(n, 1)$ were actually conformal; (cf. [30, Chapter 6]). 
Problem 10. Extend Gromov's proof to deduce the Margulis rigidity theorem [32] for other cocompact lattices.

Problem 11 (Hurder). Use Gromov's method to prove a generalized rigidity theorem: Let $\Gamma$ be a discrete subgroup of a simple Lie group $G$ which is not locally isomorphic to $\operatorname{SL}(2, \mathbb{R})$. Suppose $G$ is the algebraic hull of $\Gamma$ and for some $p>2$ the bounded cohomology group $H_{b d d}^{p}(\Gamma) \neq 0$. Then show that for all discrete actions of $\Gamma$ on a contractible homogeneous $p$-manifold $X$ with $X / \Gamma$ compact, the action of $\Gamma$ on $X$ is rigid.

Remark. If we allow in problem 11 that $X$ is an arbitrary contractible space of dimension $p$, then this is a special case of Borel's question [6]. Connes' work in [5] shows that the algebraic obstructions to rigidity (i.e. to the Kasparov reduction of the problem) vanish if $H_{b d d}^{*}(\Gamma)$ is large enough to detect all of the classes in the $K$-homology group $K_{*}(B \Gamma)$ of the classifying space $B \Gamma$. For a homogeneous space $X$, problem 11 asks whether it is just the cohomology classes in $H_{\text {bdd }}^{*}(\Gamma)$ of top dimension $=$ dimension $X$ which determine everything.

A measurable foliation $\mathscr{F}$ on a finite measure space $(X, \mu)$ is a measurable partition of $X$ into smooth submanifolds of constant dimension satisfying a local triviality condition (cf. [16] and [34]). On such $\mathscr{F}$ we can define a measurable family, $g$, of Riemannian metrics on the leaves of $\mathscr{F}$. Let $d \mu_{g}$ denote the corresponding Riemannian volume form on the leaves, and define a transverse measure $d \mu_{T}$ for $\mathscr{F}$ by setting $d \mu=d \mu_{\mathrm{g}} \times d \mu_{T}$. We will call $(X, \mathscr{F}, \mu, g)$ a measurable Riemannian foliation [16], [34].

We say that $(X, \mathscr{F}, \mu, g)$ is isomorphic to $\left(X^{\prime}, \mathscr{F}^{\prime}, \mu^{\prime}, g^{\prime}\right)$ if there is a measurable isomorphism $\Phi: X \rightarrow X^{\prime}$ which maps the leaves of $\mathscr{F}$ diffeomorphically onto the leaves of 'F', and they are isometrically isomorphic if the leaf maps are isometries. We say that $(X, \mathscr{F}, \mu, g)$ is rigid if for any $\left(X^{\prime}, \mathscr{F}^{\prime}, \mu^{\prime}, g^{\prime}\right)$ with $(X, \mathscr{F}, \mu)$ orbit equivalent to $\left(X^{\prime}, \mathscr{F}^{\prime}, \mu^{\prime}\right)$, then the foliations are isomorphic. Zimmer has shown:

Theorem [33], [34]. Let $(X, \mathscr{F}, \mu, g)$ be a measurable Riemannian foliation, and suppose that each leaf of $\mathscr{F}$ is isometric to a complete locally symmetric space of rank $\geq 2, d \mu_{T}$ is a non-atomic invariant measure for $\mathscr{F}$ with finite total mass (so $\mathscr{F}$ is type $\left.\mathrm{II}_{1}\right)$ and $\mathscr{F}$ is ergodic with respect to $\mu_{T}$. If $(X, \mathscr{F}, \mu, g)$ is orbit equivalent to another such $\left(X^{\prime}, \mathscr{F}^{\prime}, \mu^{\prime}, g^{\prime}\right)$ then they are isometrically isomorphic.

Problem 12. Extend Zimmer's theorem above to conclude that such $(X, \mathscr{F}, \mu, g)$ are actually rigid in the broader sense defined above.

Problem 13. Let $(X, \mathscr{F}, \mu, g)$ be a measurable Riemannian foliation, and assume the transverse measure $d \mu_{T}$ is not invariant. Find additional hypotheses on $(X, \mathscr{F}, \mu, g)$ which will guarantee that the foliation is rigid in either of the above senses. Zimmer has given one set of sufficient additional conditions in $[32, \S 7]$. Note that the above theorem is false without the hypothesis $d \mu_{T}$ is invariant and no additional hypotheses are made.

A foliation $\mathscr{F}$ on $(X, \mu)$ defines an equivalence relation $\mathscr{R}(\mathscr{F}) \subset \boldsymbol{X} \times \boldsymbol{X}$, where $(x, y) \in \mathscr{R}(\mathscr{F})$ iff $x$ and $y$ are on the same leaf. A cocycle $\phi$ on $\mathscr{F}$ is a measurable 
map $\phi: \mathscr{R}(\mathscr{F}) \rightarrow G$ such that $\phi(x, z)=\phi(x, y) \phi(y, z)$ for $(x, y),(y, z) \in \mathscr{R}(\mathscr{F})$, where $G$ is a Polish group.

Problem 14. (Generalized super-rigidity [32].) Find conditions on a Riemannian foliation $(X, \mathscr{F}, \mu, g)$ which are sufficient to allow a good classification of the cocycles over $\mathscr{F}$ (up to cohomology).

Problem 15 (Hurder). Let $(X, \mathscr{F}, \mu)$ and $\left(X^{\prime}, \mathscr{F}^{\prime}, \mu^{\prime}\right)$ be measurable foliations, and suppose there are given cocycles $\phi: \mathscr{R}(\mathscr{F}) \rightarrow G$ and $\psi^{\prime}: \mathscr{R}\left(\mathscr{F}^{\prime}\right) \rightarrow G$ whose algebraic hull [32] is a simple Lie group $G$ of rank $\geq 2$. Let $\Phi:(X, \mathscr{F}, \mu) \rightarrow\left(X^{\prime}, \mathscr{F}^{\prime}, \mu^{\prime}\right)$ be an orbit equivalence such that $\Phi^{*}\left(\phi^{\prime}\right)$ is cohomologous to $\phi$. If almost every leaf of $\mathscr{F}$ and $\mathscr{F}^{\prime}$ is contractible, and $\mathscr{F}$ and $\mathscr{F}^{\prime}$ are irreducible in an appropriate sense, show that $(X, \mathscr{F}, \mu)$ is isomorphic to $\left(X^{\prime}, \mathscr{F}^{\prime}, \mu^{\prime}\right)$.

Remark. For a smooth foliation of a compact manifold, the results of [16] imply that $\mathscr{F}$ will have a cocycle $\phi$ as in problem 15 whenever $\mathscr{F}$ has a non-vanishing higher degree residual secondary class. The above problems 14 and 15 are the key steps towards showing that such foliations must be rigid.

4. Rigidity theorems for cocycles are very important for the proofs of the geometric rigidity theorems of Margulis and Zimmer. Cocycles arise in many geometric contexts, and these contexts also suggest corresponding rigidity problems.

Problem 16 (Katok). Let $v$ be a $C^{\infty}$-vector field on a compact manifold $M$. We say that $v$ is $C^{\infty}$-rigid if for all smooth functions $\rho>0$ on $M$, the flow of the vector field $\rho \cdot v$ is $C^{\infty}$-conjugate to the flow of $v\left(\left[18\right.\right.$, Chapter 10]). Describe all $C^{\infty}$-rigid flows on a given manifold.

Kolmogorov [20] shows that the flow on $\mathrm{T}^{2}$ with irrational rotation angle $\theta$, where $\theta$ satisfies an arithmetic condition, is $C^{\infty}$-rigid. What flows on $T^{n}$ are $C^{\infty}$-rigid?

There is a discrete version of problem 16 in terms of cocycles:

Problem 17 (Katok). Let $f: N \rightarrow N$ be a $C^{\infty}$-diffeomorphism. We say condition (*) holds for $f$ if every smooth function on $N$ is cohomologous to a constant. That is:

(*) For each $C^{\infty}$-function $\varphi: N \rightarrow \mathbb{R}$, there exists a smooth function $h=h_{\varphi}: N \rightarrow \mathbb{R}$ and a constant $\varphi_{0}$ so that

$$
\varphi(x)=\varphi_{0}+h(f(x))-h(x) \quad \text { for all } x \in N
$$

For a given manifold $N$, what diffeomorphisms of $N$ satisfy $(*)$ ?

The irrational rotation of the circle $S^{\mathbf{1}}$ by angle $\theta$ satisfies (*) if $\theta$ has at most polynomial approximation speed by rationals [20], [18].

The connection between the two problems is via the suspension construction: Given $f: N \rightarrow N$, define a new manifold $M=N \times \mathbb{R} /(x, r) \sim(f(x), r+1)$. The unit tangents to the lines $\{x\} \times \mathbb{R}$ define a flow $v$ on $M$. A solution to problem 17 for $N$ and $f$ then yields a solution to problem 16 for $M$ and $v$.

Remark (Katok). If $f: N \rightarrow N$ satisfies condition (*) then $f$ is strictly ergodic. There are obstructions, in the form of invariant distributions on $N$, which must be zero for a solution to $(*)$ to exist. In many cases it suffices to consider only invariant measures (e.g. [13]). On the other hand, as it is shown in [18, § 10.5] for a strictly 
ergodic affine map of the 2-torus $\mathbb{T}^{2}$, there is a countable family of invariant distributions which are not measures and which completely determine whether one can solve the cocycle equation (*).

Problem 18 (Katok). Let $\theta$ be an irrational number with at most polynomial approximation speed by rationals, and $R_{\theta}$ the corresponding rotation of $S^{1}$. Does the $C^{\infty}$-cocycle equation with values in $\operatorname{GL}(n, \mathbb{R})$ for $R_{\theta}$ always have a solution? That is, prove:

$\left(*_{n}\right)$ For each $C^{\infty}$-function $\varphi: S^{1} \rightarrow G L(n, \mathbb{R})$, there exists a smooth function $h=h_{\varphi}: S^{1} \rightarrow \mathrm{GL}(n, \mathbb{R})$ and a constant $\varphi_{0} \in \mathrm{GL}(n, \mathbb{R})$ so that

$$
\varphi(x)=h\left(R_{\theta} x\right) \cdot \varphi_{0} \cdot h(x)^{-1} \quad \text { for all } x \in S^{1} .
$$

5. For a given $\Gamma$ and Lie group $G$, the rigidity of $G$-cocycles over all possible finite-measure-preserving ergodic actions of $\Gamma$ is an extremely strong condition on $(\Gamma, G)$. Zimmer has proved this for the case when $\Gamma$ is a Kazhdan group and $G$ is real algebraic [37].

For a soft group $\Gamma$, rigidity for cocycles over $\Gamma$ cannot be expected. This is especially true for $\Gamma$ amenable. The surprising fact is that $\varepsilon$-rigidity can often be proved, and this property extends to the cases of cocycles over foliations and groupoids. The first seeds of $\varepsilon$-rigidity appeared in the proofs of the Pesin theory of dynamical systems [22], [23], and there are now numerous open questions.

Let $\Gamma$ be a finitely generated group, with generators $\left\{\gamma_{1}, \ldots, \gamma_{n}\right\},(X, \mu)$ a standard measure space with probability measure $\mu$, and suppose $\Gamma$ acts ergodically on $X$ preserving $\mu$. A cocycle $\Phi: \Gamma \times X \rightarrow G$ is $\varepsilon$-rigid if there exists a homomorphism $\phi_{0}: \Gamma \rightarrow G$ such that for all $\varepsilon>0$, there is a cocycle $\phi_{\varepsilon}$ cohomologous to $\phi$ such that

$$
\left\|\phi_{\varepsilon}\left(\gamma_{i}, x\right) \cdot \phi_{0}\left(\gamma_{i}\right)^{-1}\right\|_{G}<\varepsilon \quad \text { for } 1 \leq i \leq n .
$$

Problem 19 (Katok). Find conditions on the group $\Gamma$, the action $\Gamma \times X \rightarrow X$ and the cocycle $\phi$ which implies that $\phi$ is $\varepsilon$-rigid.

Remarks. (A) For $\Gamma=\mathbb{Z}$ and $\phi: \Gamma \times X \rightarrow G$ tempered ([16] and see below; here we mean $\left\|\phi\left(\gamma_{i}, x\right)\right\|_{G}<k$ for some $k$ and all $\left.1 \leq i \leq n\right)$ the Oseledec theorem [22] implies $\phi$ is always $\varepsilon$-rigid for $G=\mathbb{R}^{N}$. In this context, the $\varepsilon$-rigidity of $\phi$ is a refined form of the Birkhoff ergodic theorem. More generally, problem 19 asks for which groups $\Gamma$ and classes of cocycles over $\Gamma$ does there exist an ergodic theorem?

(B) For $\Gamma=\mathbb{Z}^{n}$, every $L^{p}$-cocycle for $p>n$ has the $\varepsilon$-rigidity property [19], but there are $L^{n}$-cocycles which are not $\varepsilon$-rigid. (The difficulty which arises for $p \leq n$ is in obtaining asymptotic estimates on the cocycle.)

(C) For $\Gamma$ nilpotent, every tempered cocycle has the $\varepsilon$-rigidity property [19].

Problem 20. For $\Gamma$ of polynomial growth rate $p_{0}$, show that every $L^{p}$-cocycle over $\Gamma$ for $p>p_{0}$ has the $\varepsilon$-rigidity property. For $\Gamma$ of subexponential growth, show that every tempered cocycle over $\Gamma$ is $\varepsilon$-rigid. Can anything be said for $\Gamma$ amenable?

A concept closely related to $\varepsilon$-rigidity is the $\varepsilon$-classification property, which is in fact a key ingredient in establishing $\varepsilon$-rigidity. We formulate this in a very general context.

Let $(X, \mu)$ be a measure space and $R \subset X \times X$ a measurable equivalence relation on $X$. Let $d: R \rightarrow \mathbb{R}^{+}$be a measurable family of metrics on the orbits of $R: d(x, y)=$ 
$d(y, x) \geq 0$ for $(x, y) \in R$ and is measurable in $x$ and $y$. We say $(X, R, d)$ is a metric equivalence relation of exponential type [16] if each ball

$$
B(x, r)=\{(x, y) \in R \mid d(x, y)<r\}
$$

has at most finitely many points, and the limit

$$
c(x)=\limsup _{r \rightarrow \infty} \frac{\log \# B(x, r)}{r}<\infty \quad \text { a.e. } x \in X .
$$

We say $(X, R, d)$ has subexponential type if $c(x)=0$ for a.e. $x \in X$. This means the number of elements in a.e. ball along the orbits of $R$ grows subexponentially with the radius.

Let $G$ be a Lie group with a left-invariant Riemannian metric, and let \|\|$_{G}$ denote the corresponding distance to the identity element. A cocycle $\phi: R \rightarrow G$ is $\varepsilon$-tempered if

$$
\|\phi(x, y)\|_{G}<\varepsilon \cdot d(x, y) \quad \text { a.e. }(x, y) \in R .
$$

We say $\phi$ has moderate growth if

$$
\limsup _{d(x, y) \rightarrow \infty} \frac{\log \|\phi(x, y)\|_{G}}{d(x, y)}=0 \quad \text { a.e. } x \in X .
$$

A Lie group $G$ has the $\varepsilon$-classification property [19] if for every metric equivalence relation $(X, R, d)$ with subexponential type, for every $G$-cocycle $\phi$ over $R$ with moderate growth and for every $\varepsilon>0$, there exists an $\varepsilon$-tempered cocycle $\phi_{\varepsilon}: R \rightarrow G$ which is cohomologous to $\phi$.

Problem 21. Which Lie groups have the $\varepsilon$-classification property?

Remarks. $\mathbb{R}^{n}$ has the $\varepsilon$-classification property for all $n \geq 1$ by theorem 3.2 of [16]. A Lie group $G$ has the cone property if the set $\left\{g^{*} g \mid g \in G\right\}$ is a cone. That is for all $\alpha, \beta>0$ and $g_{1}, g_{2} \in G$, we can find $g_{3} \in G$ so that

$$
\alpha \cdot g_{1}^{*} g_{1}+\beta \cdot g_{2}^{*} g_{2}=g_{3}^{*} g_{3} \text {. }
$$

The groups $G=\mathrm{GL}(n, \mathbb{R}), \mathrm{GL}(n, \mathbb{C}), \mathrm{SO}^{*}(4 n), \mathbb{R}^{n}, \mathrm{SO}(n, 1)$ and one of the real forms of $E_{6}$ all have the cone property.

Theorem 3.2 of [16] actually shows that if $G$ has the cone property, then $G$ has the $\varepsilon$-classification property.

A maximal amenable subgroup of $\mathrm{GL}(n, \mathbb{R})$ or $\mathrm{GL}(n, \mathbb{C})$ has the $\varepsilon$-classification property [16].

No examples are known of either nilpotent, solvable or simple Lie groups which do not have the $\varepsilon$-classification property. The case for $G$ nilpotent is the first important case to consider.

6. Let $X$ be a metric space with metric $d: X \times X \rightarrow \mathbb{R}^{+}$. A continuous action of a group $\Gamma$ on $X$ is distal if for all $x \neq y$ in $X$,

$$
\underset{\gamma \in \Gamma}{\operatorname{infimum}} d(\gamma x, \gamma y)>0 .
$$

The action is minimal if for each $x \in X$, the orbit $\Gamma x$ is dense in $X$. Generalizing a fundamental theorem of Furstenberg, M. Rees showed in her thesis [26] that a 
minimal distal action on a compact manifold $X$ is always obtained, up to homeomorphism, by a finite number of isometric extensions [9], where the structure groups involved are compact Lie groups, but the fibrations need not be smooth. An extensive analysis of minimal actions is given in I. Bronstein's book [38]. When $\Gamma$ is a Kazhdan group, Zimmer has proved a related result.

THEOREM [37]. Let $\Gamma$ be a Kazhdan group acting smoothly on a compact manifold $X$ and which preserves a smooth distal structure [37] on TX. Then $\Gamma$ acts via isometries on $X$ for some smooth metric on $X$.

Problem 22 (Zimmer). Suppose $\Gamma$ is a Kazhdan group, $X$ a compact manifold and suppose $\Gamma$ acts smoothly, minimally and distally on $X$. Show that $\Gamma$ preserves a smooth measure on $X$ and thus acts isometrically.

For $\Gamma$ a soft group, the conclusion of problem 22 is known to be false. The simplest counterexample to this weaker requirement is to take a diffeomorphism $f: S^{\mathbf{1}} \rightarrow S^{\mathbf{1}}$ with irrational rotation angle and so that $f$ is not $C^{1}$-conjugate to a rotation of $S^{1}$. This defines a distal action $\mathbb{Z} \times S^{1} \rightarrow S^{1}$ which is minimal, but admits no absolutely continuous invariant measure.

The concept of $\varepsilon$-classification provides a substitute for the above problem. Let $\Gamma$ be a finitely generated group with generators $\left\{\gamma_{1}, \cdots, \gamma_{d}\right\}$. Assume that $\Gamma$ acts smoothly on a compact $n$-dimensional manifold $M$. Choose a measurable framing of $T M$ and let

$$
d \gamma: \Gamma \times M \rightarrow G L(n, \mathbb{R})
$$

be the associated Jacobian cocycle of the action. The additive Radon-Nikodyn cocycle is obtained from $d \gamma$ by setting

$$
d \nu=\log (\operatorname{det}(d \gamma)): \Gamma \times M \rightarrow \mathbb{R}^{*} .
$$

Problem 23 (Hurder). Let $\Gamma$ act smoothly on $M$ as above and suppose the action is minimal and distal. Show the cocycle $d \nu$ has the $\varepsilon$-classification property. That is, for all $\varepsilon>0$ there is a cocycle $\phi_{\varepsilon}$ cohomologous to $d \nu$ with

$$
\left|\phi_{\varepsilon}\left(\gamma_{i}, x\right)\right|<\varepsilon \quad \text { for all } 1 \leq i \leq d \text {, a.e. } x \in M \text {. }
$$

Note that $\phi_{\varepsilon}$ is just the divergence of the $\Gamma$-action with respect to a new choice of Lebesgue density on $\Lambda^{n} T M$.

Remark. If $\Gamma$ acts equicontinuously on $M$, then lemma 4.7 and theorem 4.8 of [14] show that the conclusion of problem 23 holds.

If $\Gamma$ has subexponential growth, then for all smooth actions of $\Gamma$ on $M$ the conclusion that $d \nu$ has $\varepsilon$-classification is proved in theorem 4.10 of [15]. This suggests asking whether $\Gamma$ amenable implies that for all smooth $\Gamma$ actions, $d \nu$ has $\varepsilon$-classification?

Problem 24 (Hurder). Let $\Gamma$ act smoothly on $M$, and suppose the action is minimal and distal. Show that the cocycle $d \gamma$ is $\varepsilon$-distal: There exists a distal subgroup $D \subset G L(n, \mathbb{R})$ such that for all $\varepsilon>0$, there is a cocycle $\phi_{\varepsilon}: \Gamma \times M \rightarrow G L(n, \mathbb{R})$ cohomologous to $d \gamma$ for which

$$
\operatorname{dist}_{\mathrm{GL}(n, \mathbb{R})}\left(\phi_{\varepsilon}\left(\gamma_{i}, x\right), D\right)<\varepsilon \quad \text { all } 1 \leq i \leq d \text {, a.e. } x \in M \text {. }
$$


Remark (Katok). For $\Gamma \times M \rightarrow M$ as in problem 24 , each $\gamma \in \Gamma$ must have zero exponents on $T M$ by results in [17]. The problem is thus to obtain a field over $M$ of flags in the tangent spaces $T_{x} M$ which are 'almost invariant' under the action of $\Gamma$.

7. Let $R$ be a hyperfinite equivalence relation [7] on a measure space $(X, \mu)$. It is well-known that every cocycle $\phi: R \rightarrow G$ is then in the weak closure of the coboundaries (e.g. see [27]). The topology on cocycles is defined to be convergence in measure. If $(X, \mu, R, d)$ is a metric equivalence relation (see $\S 5$ or [16]) and $G$ is given a left-invariant norm \|\|$_{G}$, then we can define $L^{p}$-norms on the cocycles and ask whether a similar result holds.

Let $T \subset X$ be a full discrete transversal to $R,[16]$, and let $d \mu_{T}$ be the induced measure on $T$ from $\mu$. For a cocycle $\phi: R \rightarrow G$ and all $p>0$, set

$$
\|\phi\|_{p}=\left\{\int_{x \in T} \sup _{\substack{|x, y|=1 \\(x, y) \in R}}\|\phi(x, y)\|_{G}^{p} d \mu_{T}\right\}^{1 / p} .
$$

The norm $\|\phi\|_{p}$ depends on the choice of $T$ and $d$; another choice of $T$, or another choice of metric $d^{\prime}$ which is quasi-isometric [16] to $d$ will result in equivalent norms.

We say $\phi$ is in the $L^{p}$-closure of the coboundaries if for all $\varepsilon>0$, there exists a cocycle $\phi_{\varepsilon}$ cohomologous to $\phi$ with $\left\|\phi_{\varepsilon}\right\|_{p}<\varepsilon$.

Problem 25 (Hurder). Given a metric equivalence relation $(X, \mu, R, d)$ with $R$ hyperfinite, given a Lie group $G$ and fix $0<p \leq \infty$, characterize the cocycles over $R$ which are in the $L^{p}$-closure of the coboundaries.

The subspace of the cocycles over $R$ obtained by taking the $L^{p}$-closure of the coboundaries is an invariant of the quasi-isometry type of the metric $d$, and is apparently a very sensitive invariant. For example, theorem 4.10 of [15] implies that for $\Gamma$ of subexponential growth acting on a measure space $(X, \mu)$, such that the Radon-Nikodym cocycle $d \nu: \Gamma \times X \rightarrow \mathbb{P}^{+}$is tempered, then $d \nu$ has $\varepsilon$-classification. Thus, for the word metric [16] on $R=\Gamma \times X$, the cocycle $d \nu$ is in the $L^{p}$-closure of the coboundaries for all $0<p \leq \infty$. On the other hand, for $\Gamma_{g}=\pi_{1} \Sigma_{g}$ a surface group acting smoothly on $S^{1}$ via the action of $\Gamma_{g}$ on the Poincare disc and for the groupoid $R=\Gamma_{g} \times S^{1}$ with the word metric, the cocycle $d \nu: \Gamma_{g} \times S^{1} \rightarrow \mathbb{R}^{+}$is not in the $L^{p}$-closure of the coboundaries for all $p \geq 1$. (The Godbillon-Vey invariant [15] of the action is an obstruction.) The action of $\Gamma_{g}$ on $S^{1}$ is hyperfinite, so is orbit equivalent to any ergodic action of type $\mathrm{III}_{\mathbf{1}}$ on $S^{\mathbf{1}}$ by a group $\Gamma$ with subexponential growth. Thus, within a given orbit equivalence class, the property that $d \nu$ be in the $L^{P}$-closure of the coboundaries is a non-trivial invariant of the quasi-isometry type of $R$.

8. We conclude with a folklore problem from Spatzier [29], with some updated remarks:

Problem 26. What differential geometric or Riemannian properties of a foliation depend upon its ergodic theory and transverse measure theory?

Some recent results are:

(A) (Zimmer). The rigidity of negatively-curved foliations (see $\S 3$ above) [34]. 
(B) (Brooks). A leaf $L$ has iso-perimetric constant zero if and only if the essential spectrum of the leaf Laplacian contains zero [1].

(C) (Garnett). Let $\mu$ be a finite harmonic measure for $F$. A set $X \subset M$ is saturated if it is a union of leaves, and type $I$ if it has a measurable subset which intersects a.e. leaf of $X$ precisely once (a cross-section). Then for $X$ a saturated set of type I in the support of $\mu$, the $\mu$-measure of $X$ must be zero [10].

(D) (Connes). Let $F$ be a foliation by surfaces which admits a finite invariant transverse measure $\mu$. Then the average Gaussian curvature of the leaves in the support of $\mu$ is non-positive [4].

(E) (Ghys). Let $F$ be a foliation by surfaces with curvature function $k$. Let $\mu$ be any harmonic measure for $F$. (There always exists at least one harmonic measure [10].) Then $\int k d \mu \leq 0$. That is, Connes' theorem in (D) always holds for harmonic measures.

(F) (Hurder and Katok). If the equivalence relation determined by $F$ on $M$ is amenable (so hyperfinite), then all of the higher degree residual secondary characteristic classes of $F$ are zero [16].

(G) (Hurder). If all leaves of $F$ have subexponential growth, then all GodbillonVey secondary classes of $F$ are zero [15].

(H) (Zimmer). Let $F$ be a measurable Riemannian foliation with an invariant transverse measure having finite total volume. If $F$ is amenable and almost every leaf is a complete simply connected manifold of non-positive sectional curvature, then a.e. leaf of $F$ must be flat [36].

\section{REFERENCES}

[1] R. Brooks. The spectral geometry of foliations. American J. Math. 106 (1984), 1001-1012.

[2] K. Burns \& A. Katok. Manifolds with non-positive curvature. Preprint (1984), MSRI; Ergod. Th. \& Dynam. Sys. 5 (1985), 307-317.

[3] S. Cappell \& J. Shaneson. Non-linear similarity. Annals of Math. 113 (1981), 315-355.

[4] A. Connes. A survey of foliations and operator algebras. Proc. Symp. Pure Math. vol. 38 I (1981), 521-628.

[5] A. Connes. Cyclic cohomology and the transverse fundamental class of a foliation. Preprint (1984), IHES.

[6] F. T. Farrell \& W. C. Hsiang. On Novikov's conjecture for non-positively curved manifolds. Annals of Math. 113 (1981), 199-209.

[7] J. Feldman. Lectures on Orbit Equivalence. Preprint (1983), Univ. of California, Berkeley; to appear in Birkhaüser, Progress in Mathematics.

[8] J. Feldman \& D. Ornstein. Semirigidity of horocycle flows over compact surfaces of variable negative curvature. Preprint, 1984.

[9] H. Furstenberg. The structure of distal flows. Amer. J. Math. 85 (1963), 477-513.

[10] L. Garnett. Statistical properties of foliations. Springer Lect. Notes in Math. 1007 (1984), 294-299.

[11] E. Ghys. Actions localement libres du group affine. Preprint 1984.

[12] M. Gromov. Bounded cohomology. Publ. IHES 56 (1983), 6-99.

[13] V. Guillemin \& D. Kazhdan. On the cohomology of certain dynamical systems. Topology 19 (1980), 291-299 and 301-312.

[14] J. Heitsch \& S. Hurder. Secondary classes, Weil measures and geometry of foliations. J. Differential Geom. 20 (1984), 291-309.

[15] S. Hurder. Godbillon measure of amenable foliations. Preprint (1984), MSRI.

[16] S. Hurder \& A. Katok. Ergodic theory and Weil measures of foliations. Preprint (1984), MSRI. 
[17] A. Katok. Lyapunov exponents, entropy and periodic orbits for diffeomorphisms. Publ. IHES $\mathbf{5 3}$ (1980), 137-173.

[18] A. Katok. Constructions in ergodic theory. Preprint, 1983; to appear in Birkhaüser, Progress in Mathematics.

[19] A. Katok. Ergodic theory and $\varepsilon$-classification of cocycles over dynamical systems. In preparation (1984).

[20] A. N. Kolmogorov. On dynamical systems with an integral invariant on the torus. Doklady A.N. SSSR 93 (1953), 763-766; in Russian.

[21] J. Millson \& D. Johnson. Deformation spaces associated to compact hyperbolic manifolds. To appear in Discrete Groups in Geometry and Analysis, Birkhaüser, Progress in Mathematics.

[22] V. I. Oseledec. Multiplicative ergodic theorem. Lyapunov characteristic numbers for dynamical systems. Trans. Moscow Math. Soc. 19 (1968), 197-221; translated from Russian.

[23] J. B. Pesin. Characteristic exponents and smooth ergodic theory. Russian Math. Surveys 32 (1977), 55-114; translated from Russian.

[24] M. Ratner. Rigidity of horocycle flows. Annals of Math. 115 (1982), 597-614.

[25] M. Ratner. Horocycle flows, joinings and rigidity of products. Annals of Maths. 118 (1983), 277-313.

[26] M. Rees. Thesis, University of Warwick (1980).

[27] K. Schmidt. Lectures on Cocycles of Ergodic Transformation Groups. MacMillan Lectures in Mathematics. MacMillan: India (1977).

[28] S. Smale. Differentiable dynamical systems. Bull. Amer. Math. Soc. 73 (1967), 747-817.

[29] R. Spatzier. Report of special session on ergodic theory and differential geometry. A.M.S. Meeting at Amherst, October 1981. Preprint.

[30] W. Thurston. The Geometry and Topology of Three-Manifolds. Princeton University Press (to appear).

[31] D. Witte. Thesis, University of Chicago (1985).

[32] R. Zimmer. Ergodic theory, group representations, and rigidity. Bull. Amer. Math. Soc. 6 (1982), 383-416.

[33] R. Zimmer. Strong rigidity for ergodic actions of semi-simple Lie groups. Annals of Math. 112 (1980), 511-529.

[34] R. Zimmer. Ergodic theory, semisimple Lie groups and foliations by manifolds of negative curvature. Publ. IHES 56 (1982), 37-62.

[35] R. Zimmer. Volume preserving actions of lattices in semi-simple groups on compact manifolds.

[36] R. Zimmer. Curvature of leaves in amenable foliations. Amer. J. Math. 105 (1983), 1011-1022.

[37] R. Zimmer. Kazhdan groups acting on compact manifolds. To appear (1984).

[38] I. U. Bronstein. Extensions of Minimal Transformation Groups. Sijthoff and Noordhoff: Germantown, Maryland, USA and Alphen aan den Rijn, The Netherlands (1979). (Translation of 1975 Russian text, published in Kisihev.) 